\title{
Filiasparosides A-D, Cytotoxic Steroidal Saponins from the Roots of Asparagus filicinus
}

\author{
Li-Bo Zhou ${ }^{\dagger}$ Tzu-Hsuan Chen, ${ }^{\ddagger}$ Kenneth F. Bastow ${ }^{\ddagger}$ Makio Shibano, ${ }^{\ddagger}$ Kuo-Hsiung \\ Lee, ${ }^{*}+$ and Dao-Feng Chen ${ }^{*}$,
}

Department of Pharmacognosy, School of Pharmacy, Fudan University, Shanghai 200032, People's Republic of China, and Natural Products Research Laboratories, School of Pharmacy, University of North Carolina, Chapel Hill, North Carolina 27599

\section{Supporting information}

S1. HMBC spectrum of compound 1 in pyridine- $d_{5}$

S2. Detailed HMBC spectrum of compound 1 in pyridine- $d_{5}$

S3. ${ }^{1} \mathrm{H}-{ }^{1} \mathrm{H}$ COSY spectrum of compound $\mathbf{1}$ in pyridine- $d_{5}$

S4. TOCSY spectrum of compound 1 in pyridine- $d_{5}$

S5. HMBC spectrum of compound 2 in pyridine- $d_{5}$

S6. Detailed HMBC spectrum of compound 2 in pyridine- $d_{5}$

S7. ${ }^{1} \mathrm{H}-{ }^{1} \mathrm{H}$ COSY spectrum of compound 2 in pyridine- $d_{5}$

S8. TOCSY spectrum of compound 2 in pyridine- $d_{5}$

S9. HMBC spectrum of compound 3 in pyridine- $d_{5}$

S10. ${ }^{1} \mathrm{H}-{ }^{1} \mathrm{H}$ COSY spectrum of compound 3 in pyridine- $d_{5}$

S11. TOCSY spectrum of compound 3 in pyridine- $d_{5}$

S12. HMBC spectrum of compound 4 in pyridine- $d_{5}$

S13. ${ }^{1} \mathrm{H}-{ }^{1} \mathrm{H}$ COSY spectrum of compound 4 in pyridine- $d_{5}$

S14. TOCSY spectrum of compound 4 in pyridine- $d_{5}$

\footnotetext{
* To whom correspondence should be addressed. Tel: +86-21-54237453. Fax: +86-21-64170921. E-mail: dfchen@shmu.edu.cn (D.F.C.) or khlee@unc.edu (K. H. L.).

${ }^{\dagger}$ School of Pharmacy, Fudan University

$\ddagger$ Natural Products Research Laboratories, School of Pharmacy, University of North Carolina at Chapel Hill.
} 
S1. HMBC spectrum of compound $\mathbf{1}$ in pyridine- $d_{5}$

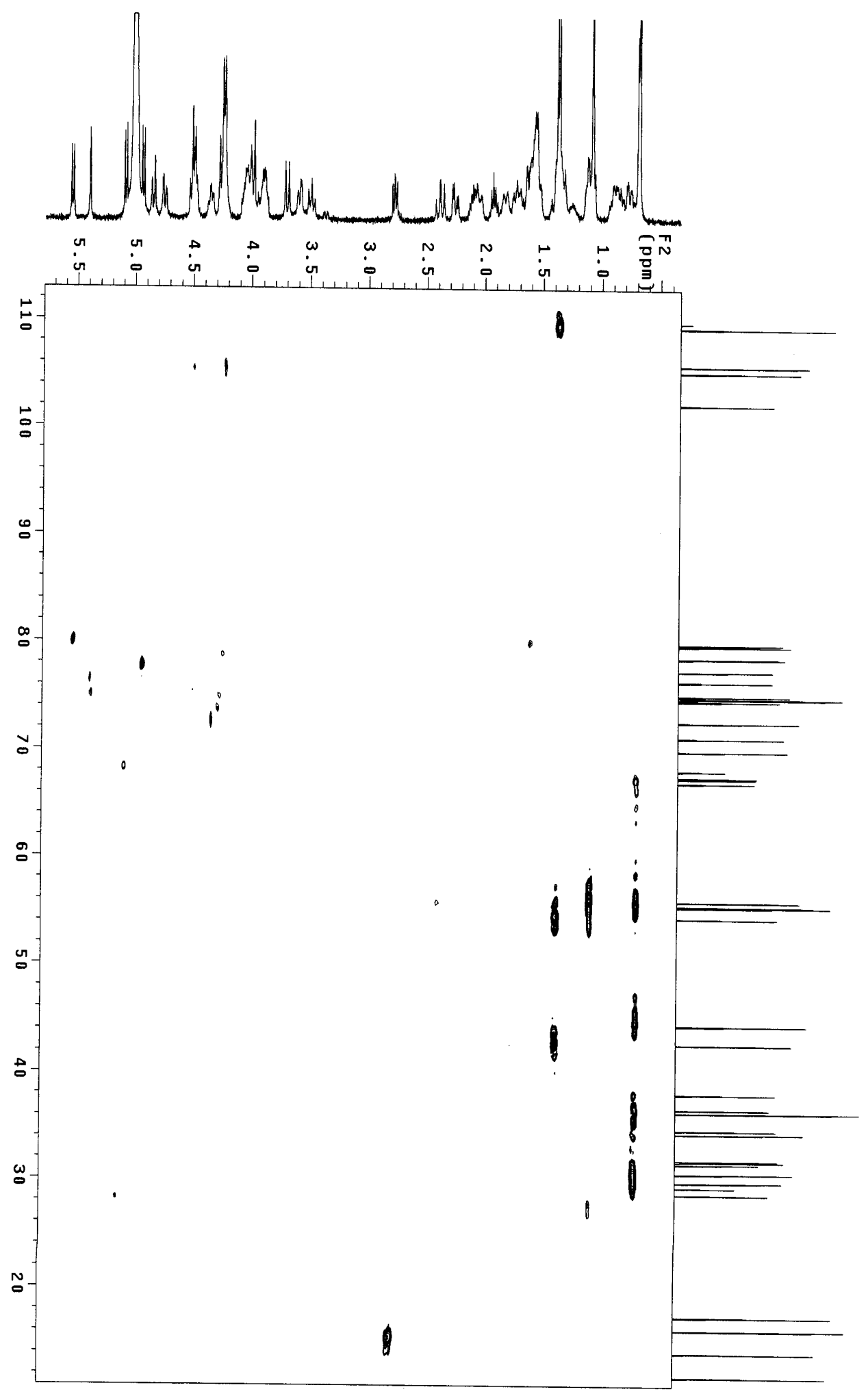


S2. Detailed HMBC spectrum of compound 1 in pyridine- $d_{5}$

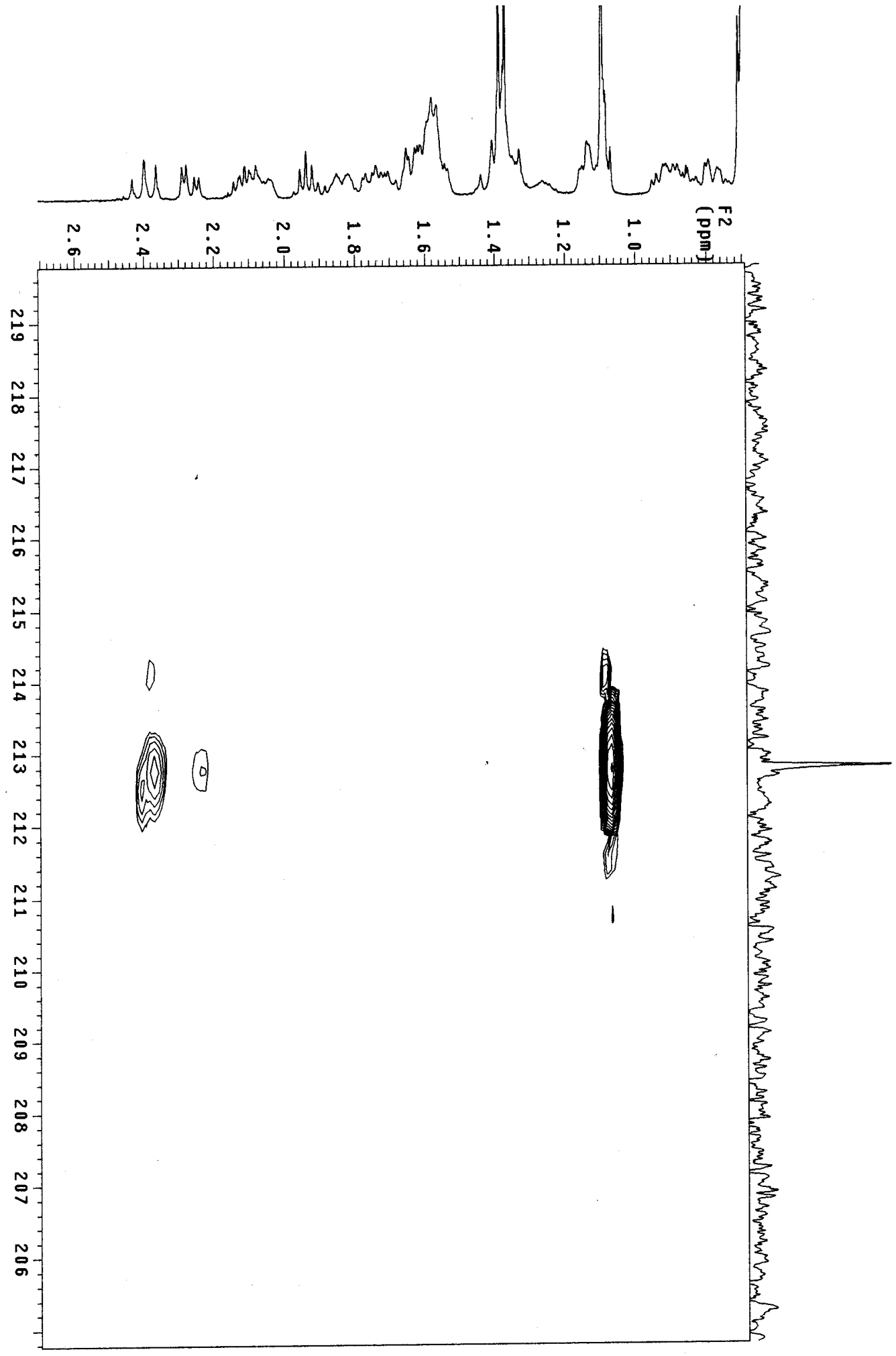


S3. ${ }^{1} \mathrm{H}-{ }^{1} \mathrm{H}$ COSY spectrum of compound $\mathbf{1}$ in pyridine- $d_{5}$

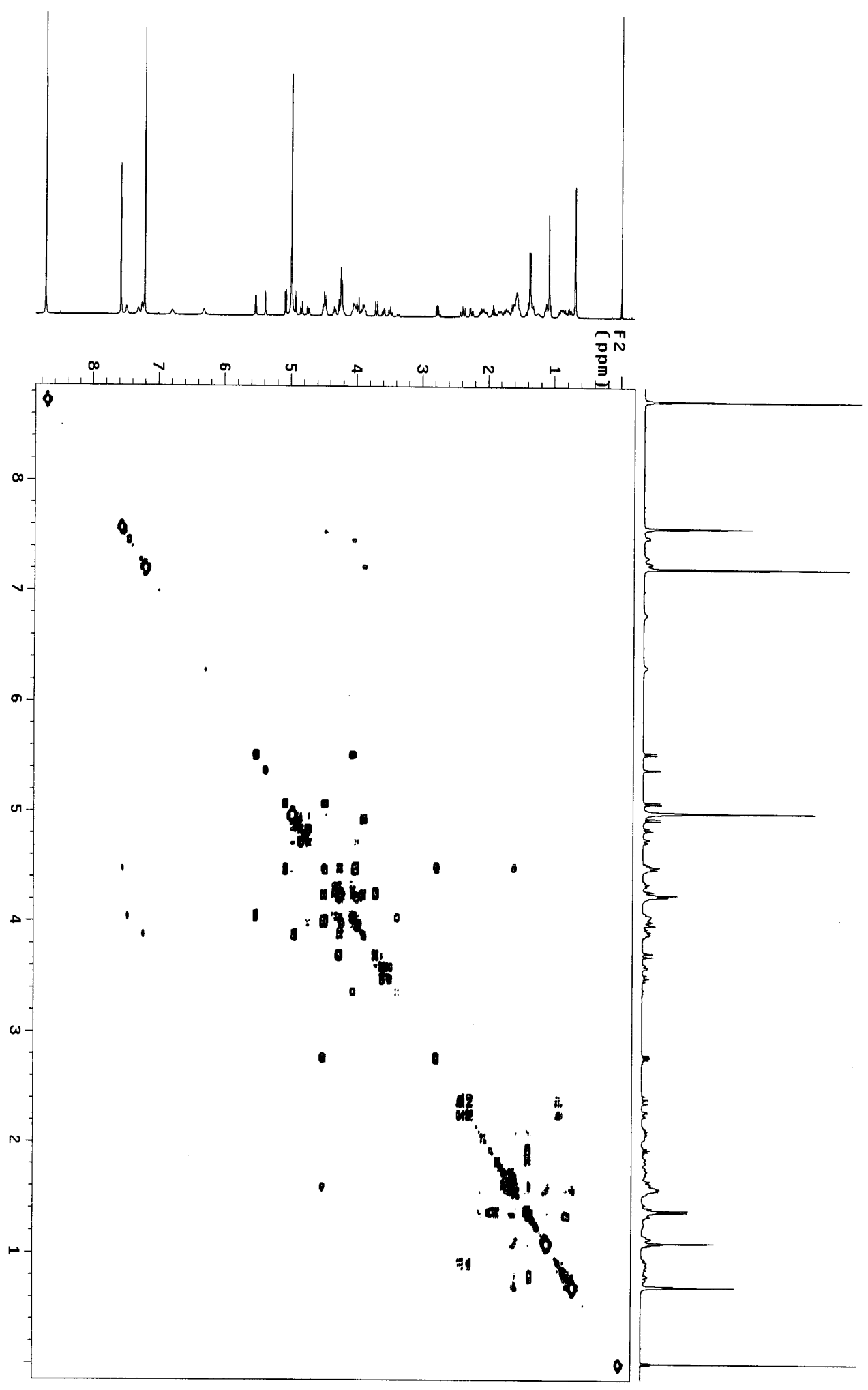


S4. TOCSY spectrum of compound 1 in pyridine- $d_{5}$

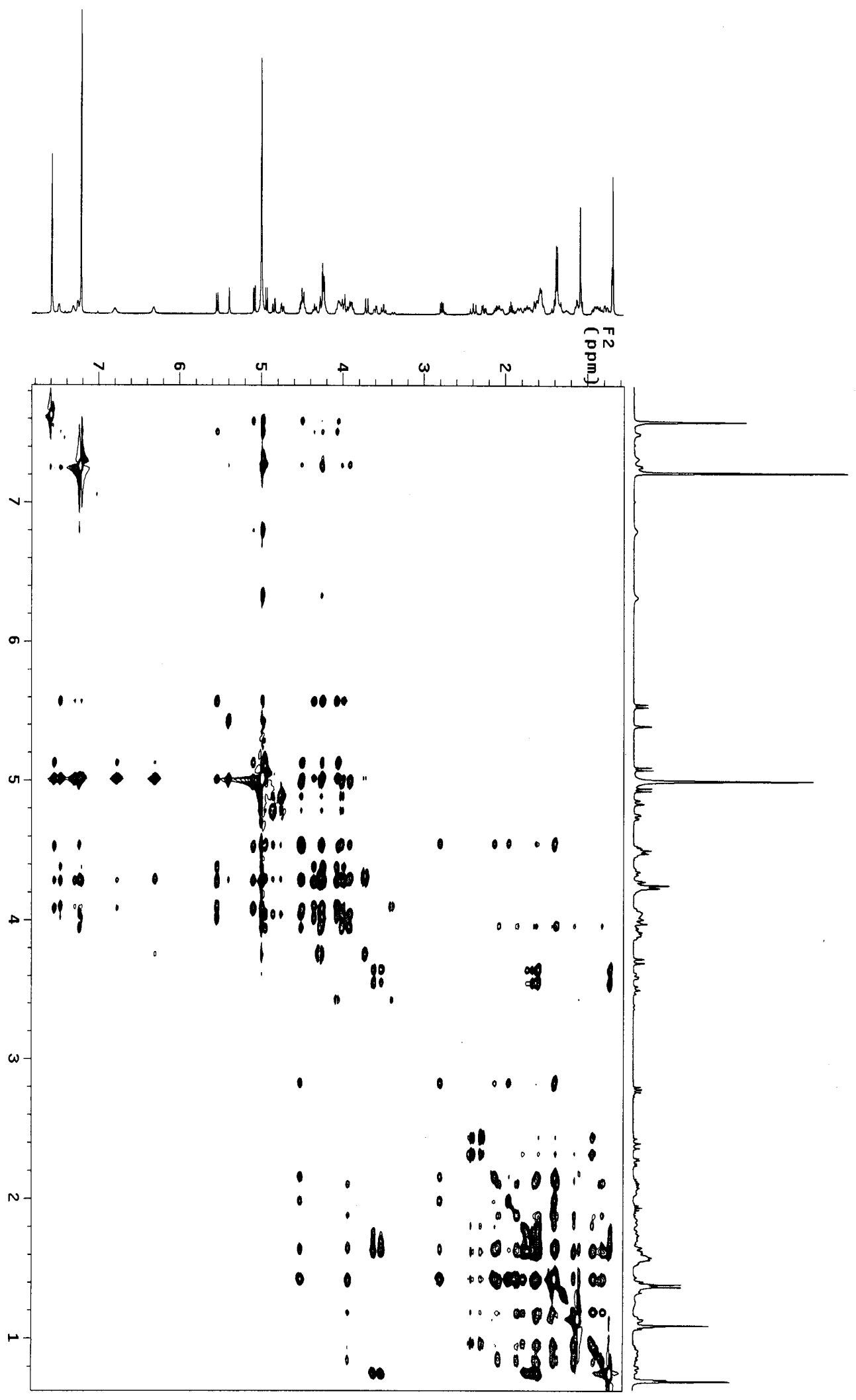


S5. HMBC spectrum of compound $\mathbf{2}$ in pyridine- $d_{5}$

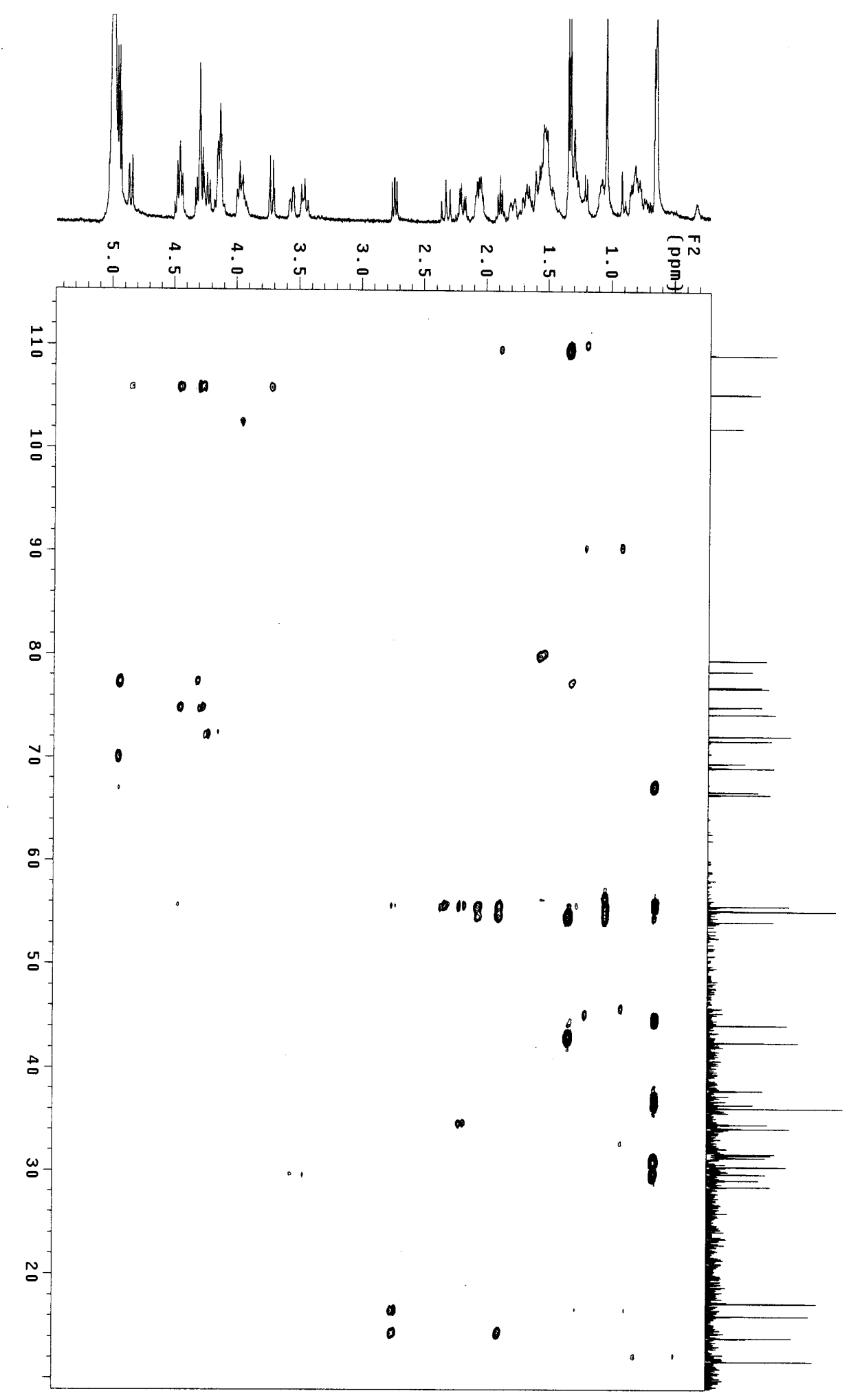


S6. Detailed HMBC spectrum of compound 2 in pyridine- $d_{5}$

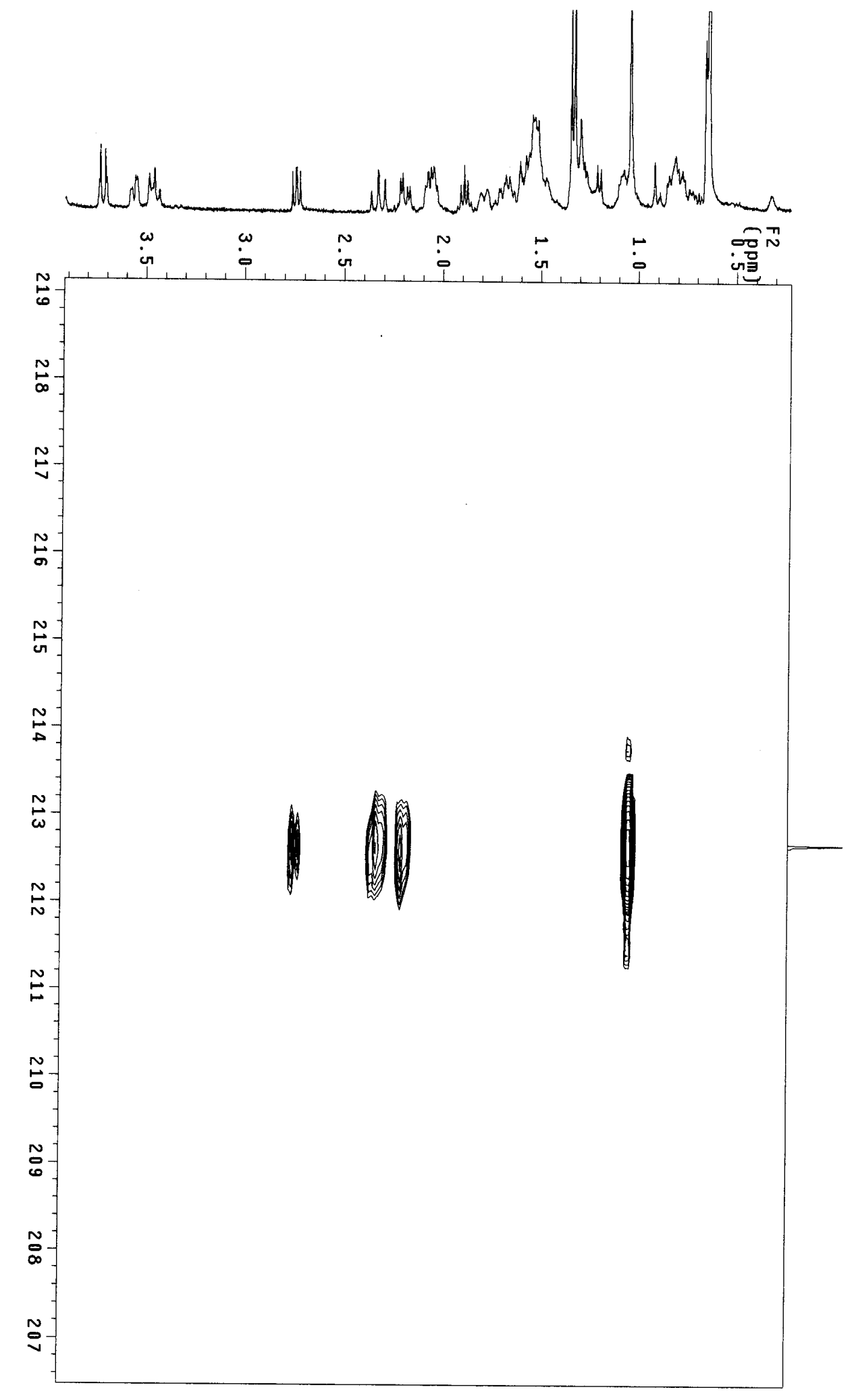


S7. ${ }^{1} \mathrm{H}-{ }^{1} \mathrm{H}$ COSY spectrum of compound $\mathbf{2}$ in pyridine- $d_{5}$

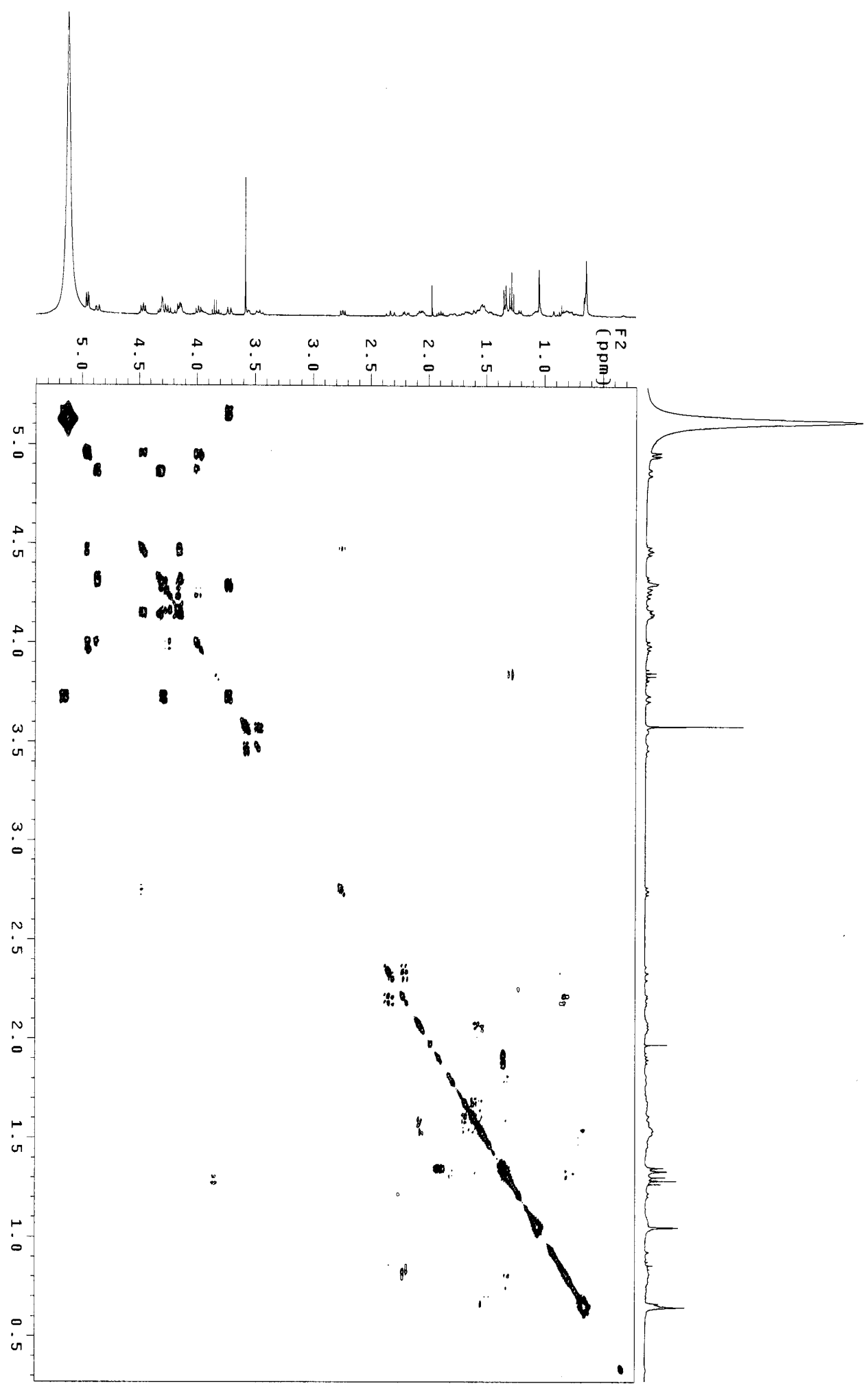


S8. TOCSY spectrum of compound 2 in pyridine- $d_{5}$

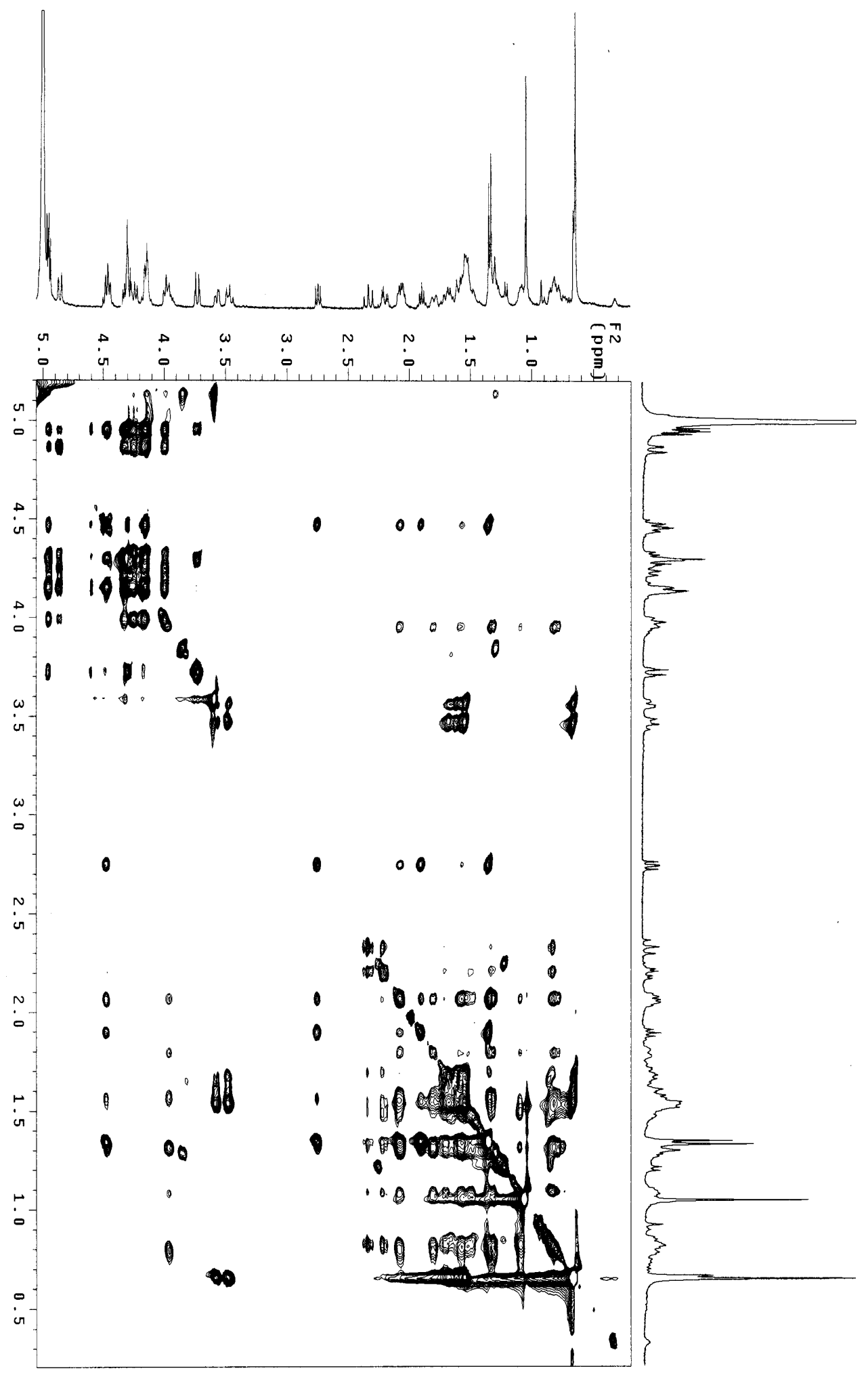


S9. HMBC spectrum of compound $\mathbf{3}$ in pyridine- $d_{5}$

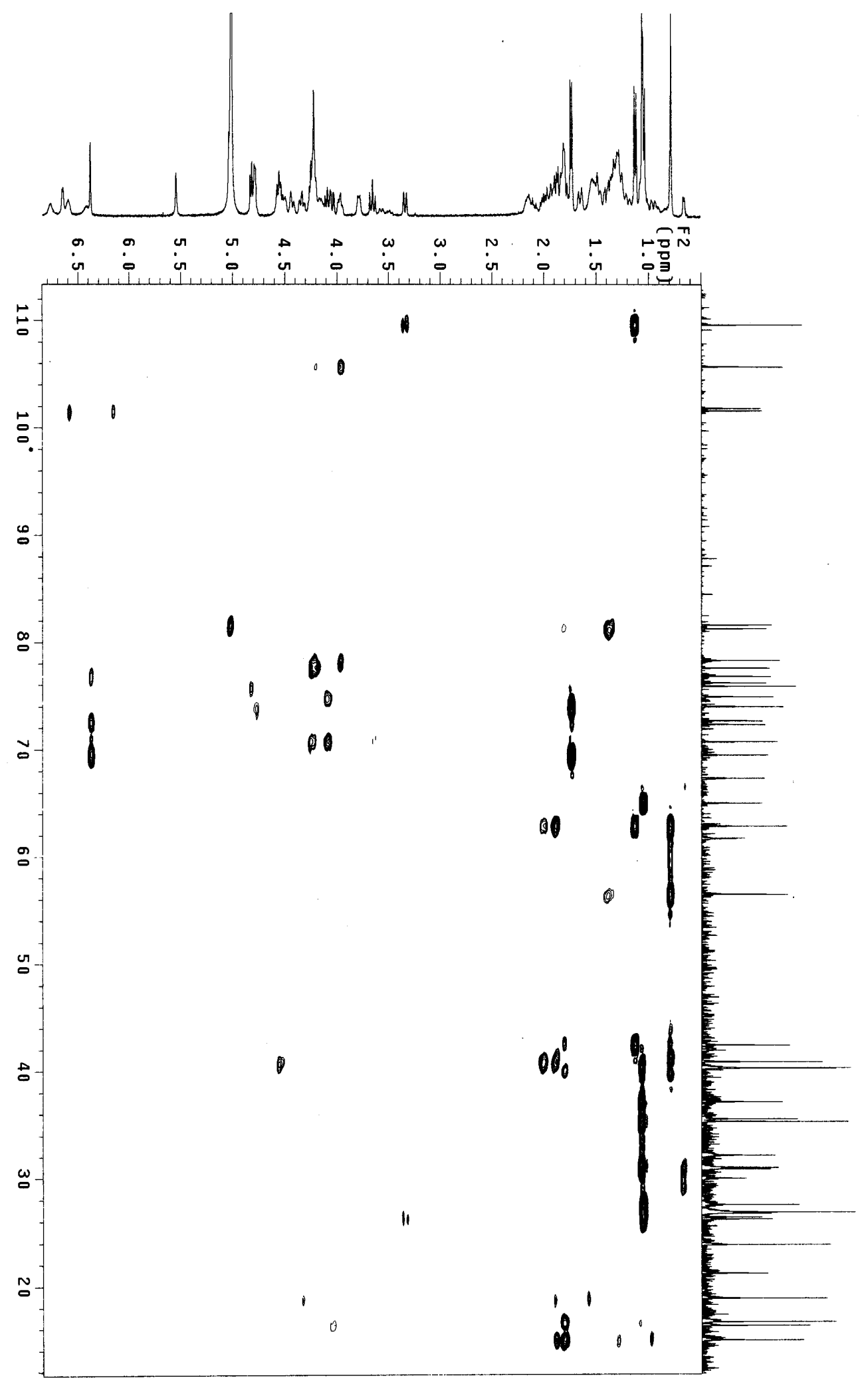


S10. ${ }^{1} \mathrm{H}-{ }^{1} \mathrm{H}$ COSY spectrum of compound 3 in pyridine- $d_{5}$

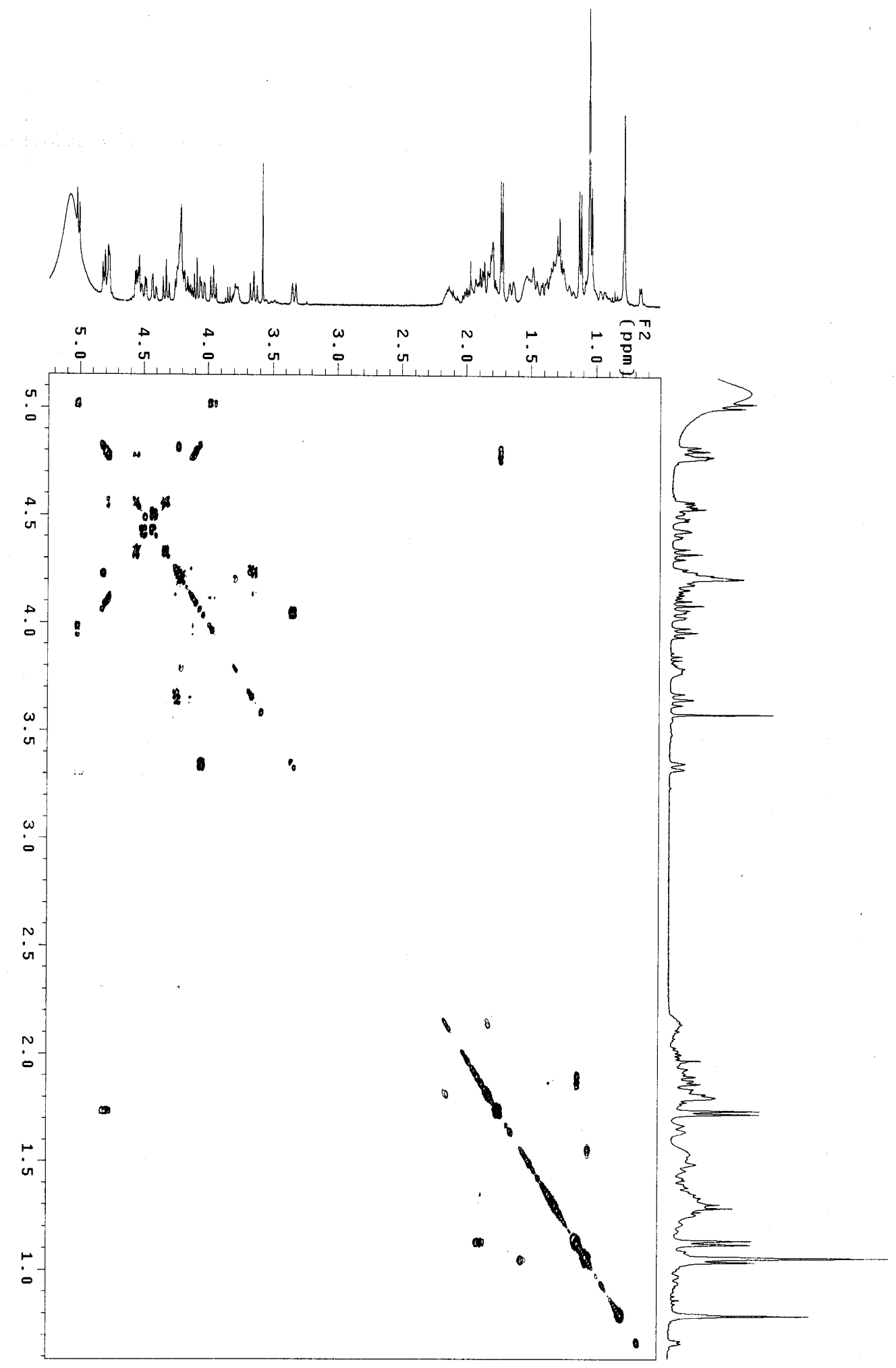


S11. TOCSY spectrum of compound 3 in pyridine- $d_{5}$

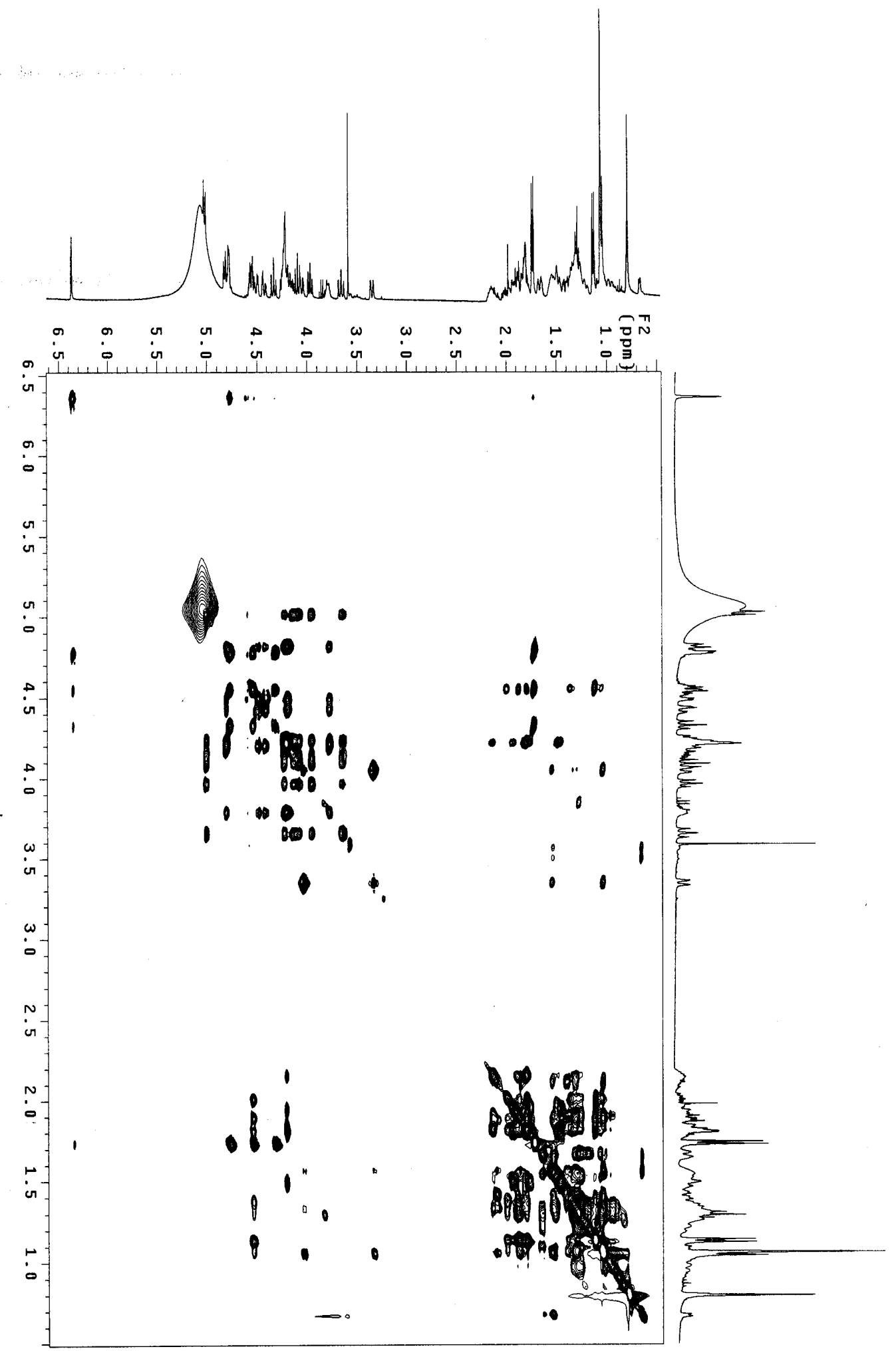


S12. HMBC spectrum of compound 4 in pyridine- $d_{5}$

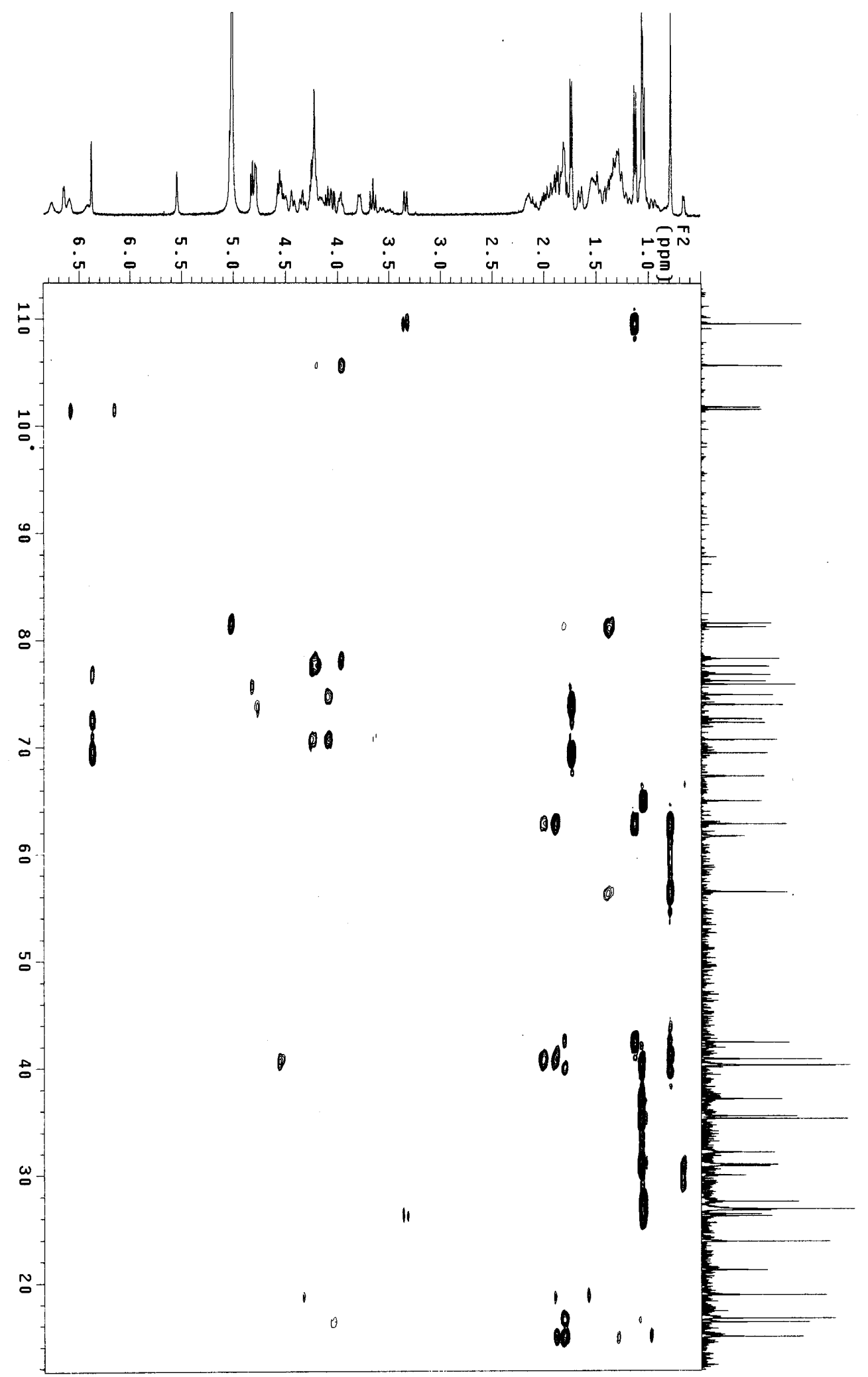


S13. ${ }^{1} \mathrm{H}-{ }^{1} \mathrm{H}$ COSY spectrum of compound 4 in pyridine- $d_{5}$

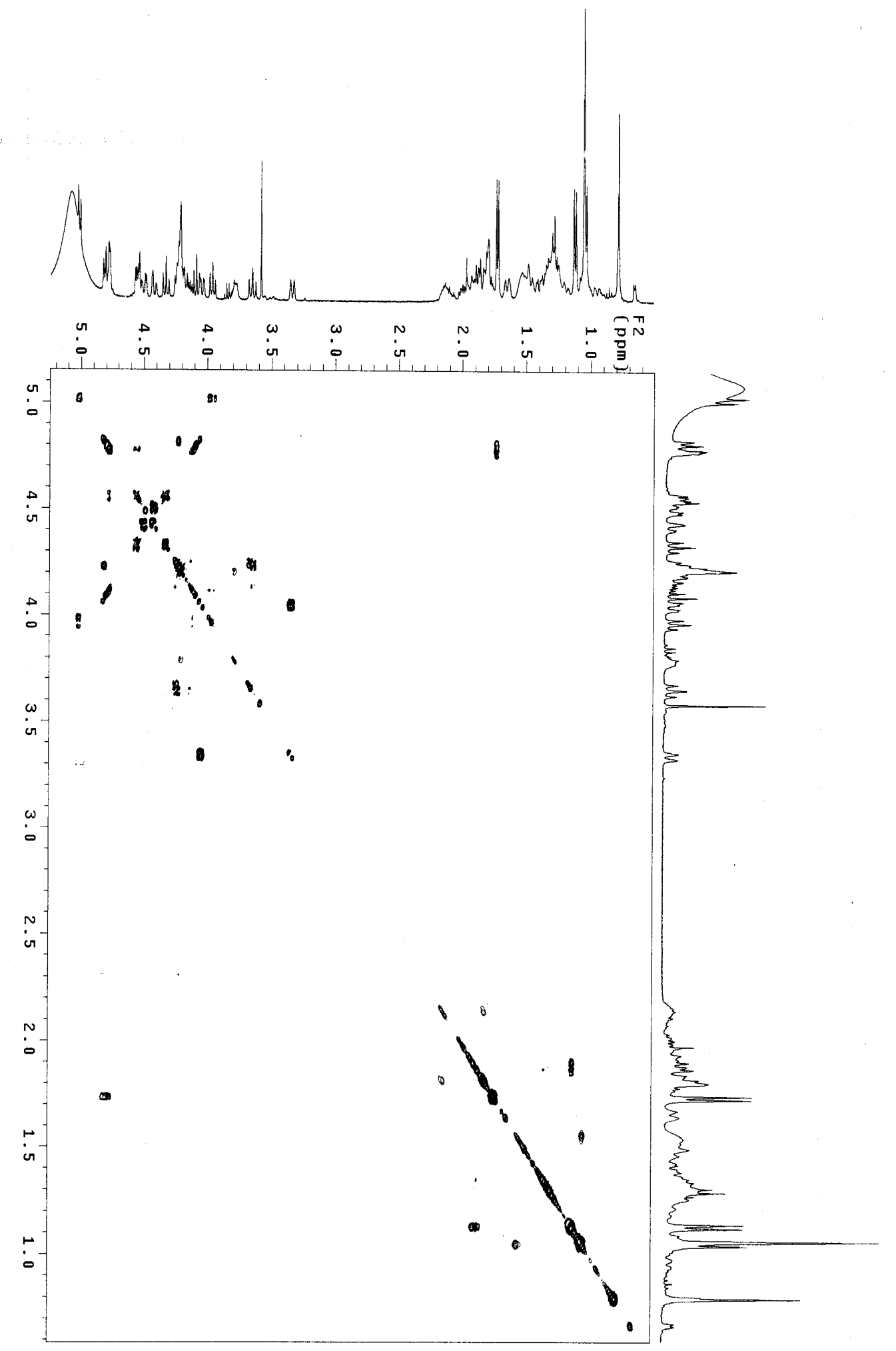


S14. TOCSY spectrum of compound 4 in pyridine- $d_{5}$

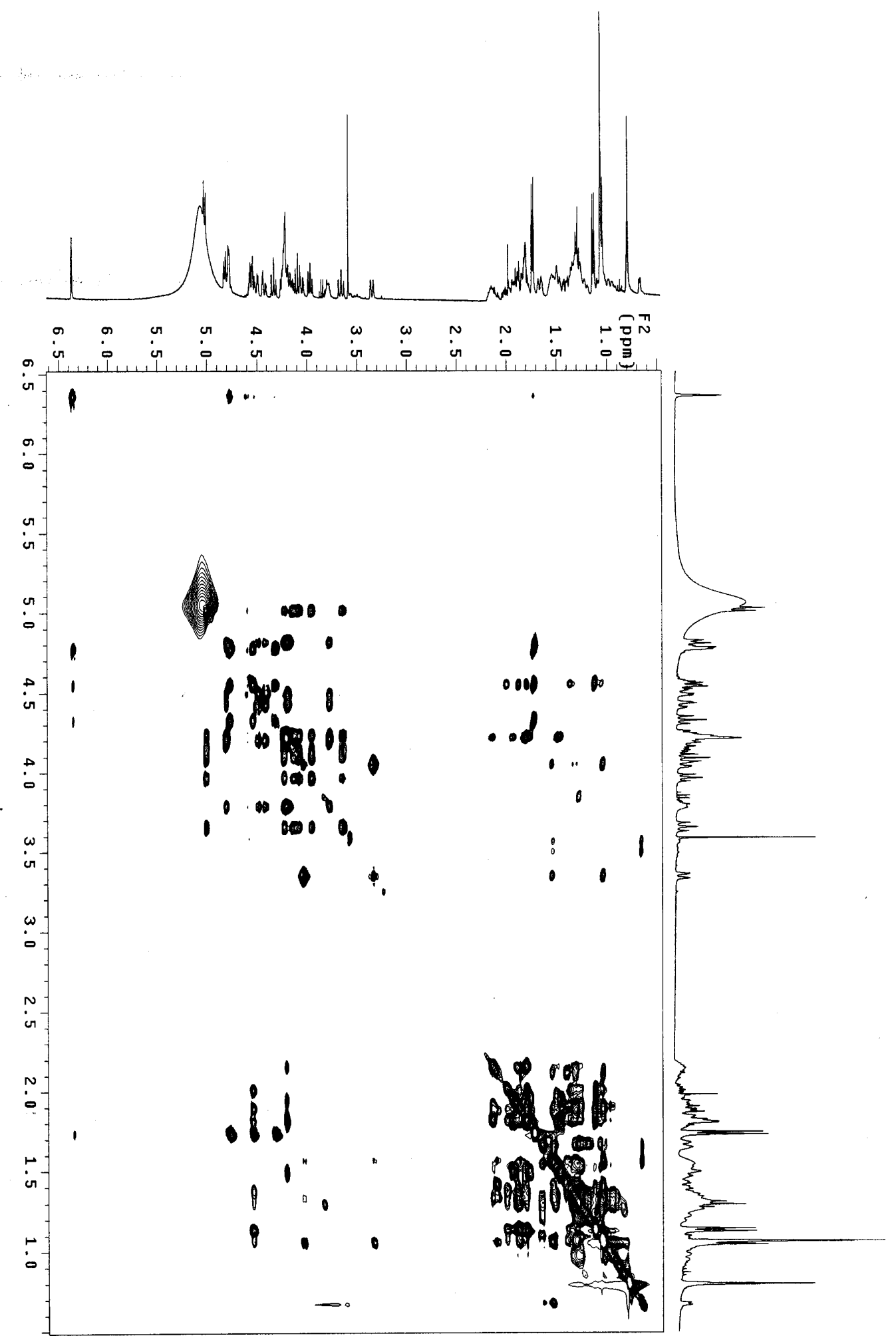

